\title{
Controlled excitation of electromagnetic band-gap line and point defect modes at microwave frequencies
}

\author{
M. Schuster ${ }^{\mathrm{a})}$ and N. Klein \\ Forschungszentrum Jülich GmbH, Institut für Schichten und Grenzflächen, D-52425 Jülich, Germany
}

(Received 4 September 2002; accepted 19 December 2002)

\begin{abstract}
We report on the controlled excitation of line and point defect modes in a two-dimensional hexagonal electromagnetic band-gap structure made of rods of dielectric material (aluminium oxide). We compared simulation performed with a numerical field simulation software and experimental measurements at microwave frequencies with regard to coupling from external waveguides to line defects and subsequent coupling to resonant modes. We observed that for a line defect in the photonic crystal the impedance matching to a waveguide is strongly dependent on the defect width. We furthermore demonstrated that the coupling to a localized defect resonance can be strongly influenced by the variation of certain single lattice elements, affecting transmission behavior and quality factor of the resonant modes. (C) 2003 American Institute of Physics.
\end{abstract}

[DOI: $10.1063 / 1.1554490]$

\section{INTRODUCTION}

It has been well known for some time that in a spatially periodic arrangement of dielectric material the propagation of electromagnetic waves is forbidden for certain frequency bands. ${ }^{1-4}$ Those frequency bands are called electromagnetic band gaps, and the dielectric arrangement is referred to as electromagnetic band-gap material (EBG) or a photonic crystal. When a point defect is introduced in the EBG lattice by local modification of the lattice parameters, localized modes with resonant frequencies inside the band gap may exist. ${ }^{5-9}$ Such localized modes have a potential to be used as a high $Q$ resonator $^{10-15}$ for various frequency ranges from the microwave range up to optical frequencies. A linearly extended defect can guide waves with high efficiency, thus allowing for the construction of beam splitters, ${ }^{16,17}$ sharp bend waveguides, ${ }^{18-22}$ and coupling structures. ${ }^{23-25}$

Photonic crystals that are periodic in two dimensions only (2D photonic crystals) can provide a band gap for every direction of propagation in the lattice plane of periodicity. ${ }^{26-28}$ It has been shown that in such a 2D type of photonic crystal the EBG behavior is different for so-called TE and TM modes, which can be distinguished by having either their electric field (TM) or magnetic field (TE) perpendicular to the 2D EBG lattice. Although they lack a band gap in the direction perpendicular to the lattice plane, 2D photonic crystals have substantial advantages in terms of compactness, stability and fabrication, which makes them attractive for microwave and optoelectronic devices. A commonly used type of $2 \mathrm{D}$ photonic lattice is the hexagonal lattice, due to its almost circular Brillouin zone and corresponding large band gaps. ${ }^{29-31}$ While the basic electrodynamic properties of photonic crystal structures have been investigated for some time and are well known, schemes for transition between an EBG structure and external microwave circuitry are still not very well developed. Although a number of experiments has been

${ }^{a)}$ Electronic mail: m.schuster@fz-juelich.de performed in the microwave frequency range, to our knowledge no special emphasis was laid on optimization of the coupling between photonic crystal waveguides and photonic crystal point defect modes. Additionally, there has been no experimental work on the optimization of impedance matching from external high frequency transmission lines, such as metal waveguides, to defect line modes in an EBG structure.

\section{NUMERICAL TOOLS AND EXPERIMENTAL SETUP}

In this article we will show and explain that the coupling from an external metal waveguide into a line defect in a two-dimensional (2D) photonic crystal can be optimized by varying the line defect size and thus matching the field distribution in the metal waveguide and line defect. Furthermore we will demonstrate, that a tuning of the coupling from a photonic crystal line defect into a resonant cavity made of an extended point defect is possible by changing the size of certain EBG lattice elements. Our work has been focused on a finite height $2 \mathrm{D}$ hexagonal photonic crystal structure made of a matrix of dielectric rods with air as a background material. The 2D EBG lattice was fully enclosed in a housing made of highly conductive copper. The rod length was selected to be $1 / 3$ of the targeted wavelength to ensure that only TM modes with their electric field along the rod axis and without nodes along this direction can propagate. The metal housing containing the EBG elements was equipped with two standard X-band waveguide ports with a width of $19.05 \mathrm{~mm}$ and a height of $9.525 \mathrm{~mm}$. Each waveguide port was terminated with a standard waveguide-to-coaxial transition to provide the connection with the measurement setup. The waveguide was operated in a mode that has its electric field in our desired direction along the rods axis, parallel to the smaller edge of the metal waveguide with no nodes along that direction. This type of mode has the lowest cutoff frequency in rectangular waveguides and is therefore dominant, which makes it very suitable for our setup. An operation in a mode with different polarization is not suitable, due to the 
relatively small height of the metal housing, which will enforce an electric field along the $y$ direction. Numerical simulations of $S$ parameters and field distributions have been performed with a commercial electromagnetic field simulation software (time domain solver of package "CST Microwave Studio" by CST Computer Simulation Technology GmbH), the band structure calculations have been performed with a transfer matrix method; the corresponding experiments have been performed using an HP 8510 network analyzer and a full two port $S$ parameter test set.

We will show a comparison between simulation and experimental results for the transmission parameter $S_{12}$ of the described 2D photonic crystal structure including various defect structures and deduce the coupling properties of the EBG structures.

The 2D photonic crystal lattice has been formed out of ceramic rods made from polycrystalline aluminum oxide $\left(\mathrm{Al}_{2} \mathrm{O}_{3}\right)$ with an isotropic dielectric constant of $\varepsilon_{r}=9.6$, loss tangent $\tan \delta=5.8 \times 10^{-5}$ at $10 \mathrm{GHz}$, radius of $2.16 \mathrm{~mm}$ and a length of $11 \mathrm{~mm}$. The ratio between rod radius $r$ and lattice constant $a$ was chosen to be 0.3 , thus yielding three photonic band gaps for TM waves. As our target frequency range was in the microwave region, we chose an absolute lattice constant of $7.2 \mathrm{~mm}$, and the resulting band gaps were located in the $\mathrm{X}$ and $\mathrm{Ku}$ band from 10 to $14.2 \mathrm{GHz}$, in the $\mathrm{K}$ band from 19.1 to $23.8 \mathrm{GHz}$ and in the Ka band from 28.3 to $32.7 \mathrm{GHz}$. In this article we will focus on the first band gap around 12 $\mathrm{GHz}$, by investigating a frequency span from 8 to $16 \mathrm{GHz}$. The axis along the metal waveguide will be referred to as $x$ axis, the one along the dielectric rods as $z$ axis and the one perpendicular to both as $y$ axis.

For each structure under investigation we will compare our results from simulation and experiment and explain its behavior in three frequency regions separately: below the band gap, in the band gap, and above the band gap.

\section{WAVEGUIDE-LINE DEFECT MATCHING}

To emphasize the effect of the introduction of a defect into the EBG lattice, we have, as a first step, calculated, and measured the behavior of the photonic crystal without any defect. The simulation of the transmission parameter $S_{21}$ of this structure is shown in Fig. 1, and the structure is shown in the inset. We can observe a transmission drop of more than $20 \mathrm{~dB}$ for frequencies between 11 and $14 \mathrm{GHz}$, corresponding to the expected band gap of the structure.

The first defect structure to be investigated is the same EBG lattice, where one line of rods had been left out to form a line defect. A comparison between simulation and experiment of $S_{21}$ is shown in Fig. 2, the structure itself is shown in the inset. We can observe that the basic features of the structure can be found in both curves, with only a slight shift in frequency. This shift was found to be caused by some error in our simulation related to the influence of the waveguide ports. At frequencies below the lower band edge, the transmission exhibits an extremely high density of structures, with an average value of around $-10 \mathrm{~dB}$. Those structures are related to the uncontrolled excitation of modes in the metal housing surrounding the photonic crystal. Above the

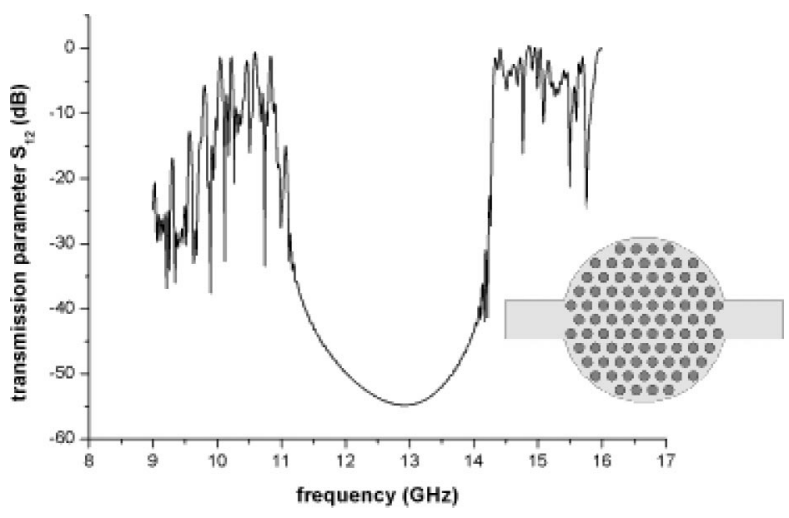

FIG. 1. 2D photonic crystal lattice without defect structures: the graph shows a simulation of the transmission parameter $S_{12}$ between 8 and $16 \mathrm{GHz}$ to indicate the transmission drop for frequencies inside the band gap. A drop of more than $20 \mathrm{~dB}$ can be observed. The assembly of the photonic crystal is shown in the inset.

lower edge of the photonic band gap around $10 \mathrm{GHz}$ the transmission drops to around $-40 \mathrm{~dB}$. The excitation of housing modes is now strongly suppressed because their existence is forbidden due to the EBG properties of the photonic crystal. As can be seen from a number of peaks inside the band gap, this suppression is not complete due to the finitesized EBG lattice, but there is no significant transmission for frequencies inside the band gap supported by those housing modes. Additionally, a broadband guiding by defect modes of the line defect does not occur. However, with increasing frequency in the band gap we observe that above a certain in-gap frequency a number of transmission peaks appears. This frequency is $13.02 \mathrm{GHz}$ in our case (peak marked with an arrow in Fig. 2). The transmission peaks are related to the excitation of standing waves in the line defect with a different number of field maxima along the defect ( $1-4$ maxima), as shown by the simulated field distribution displayed in Fig. 3. In accordance with a conventional metal waveguide the resonant frequencies $f_{n}$ were found to be determined by the

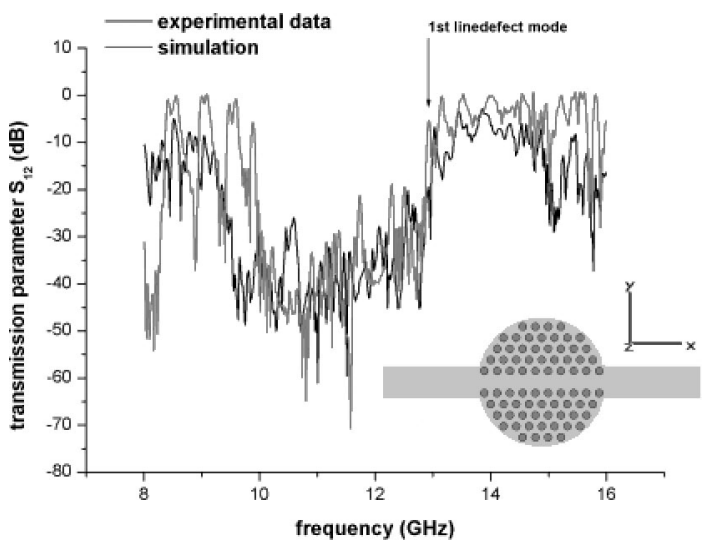

FIG. 2. One-row line defect in a 2D hexagonal photonic crystal: comparison between simulation results and experimental measurement in the microwave range from 8 to $16 \mathrm{GHz}$. The graph shows the transmission parameter $S_{12}$. The structure under investigation is shown in the inset. We can observe a high mode density below and above the band gap, a drop in transmission due to the band gap and several transmission peaks of line defect modes starting around $13 \mathrm{GHz}$. 

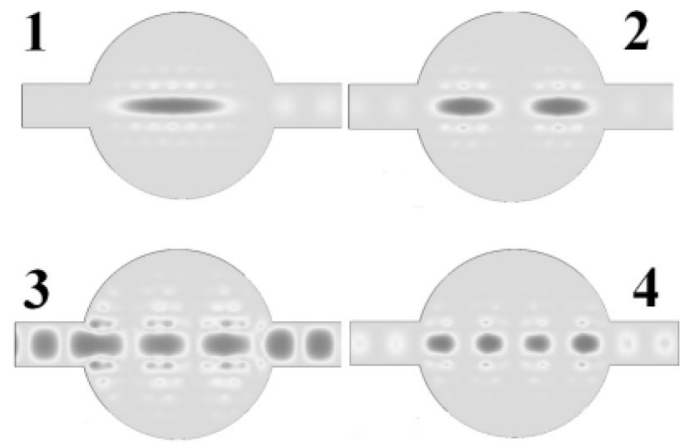

FIG. 3. Line defect modes in a one-row line defect in a 2D hexagonal photonic crystal. The shown electric field distributions (absolute values) are related to the in-band transmission peaks shown in Fig. 2. The calculated resonant frequencies are: $f_{1}=13.02 \mathrm{GHz}, f_{2}=13.44 \mathrm{GHz}, f_{3}=13.85, f_{4}$ $=14.33 \mathrm{GHz}$

cutoff frequency of the lowest order mode and the mode index $n$ (number of maxima) along the propagation direction by the equation:

$$
f_{n}=\frac{c}{2} \sqrt{f_{c}^{2}+\left(\frac{n}{L}\right)^{2}}
$$

From a fit of Eq. (1) the cutoff frequency of our line defect waveguide was determined to be $12.85 \mathrm{GHz}$. The existence of a cutoff frequency within the band gap is consistent with the flat dispersion curves of similar line defect wavguides reported in the literature. ${ }^{32-34}$ We have calculated the band structure of the lattice with this type of line defect and as can be seen from Fig. 4, we observe the existence of a defect mode inside the band gap above a certain cutoff frequency (dotted line in Fig. 4).

Overall, it can be seen clearly that there is no broadband matching between traveling modes in the metal waveguide and the one-row photonic crystal line defect. This lack of matching can be explained from a geometrical point of view, looking at the transverse field distributions of traveling waves in the metal and defect waveguides. In $y$ direction the

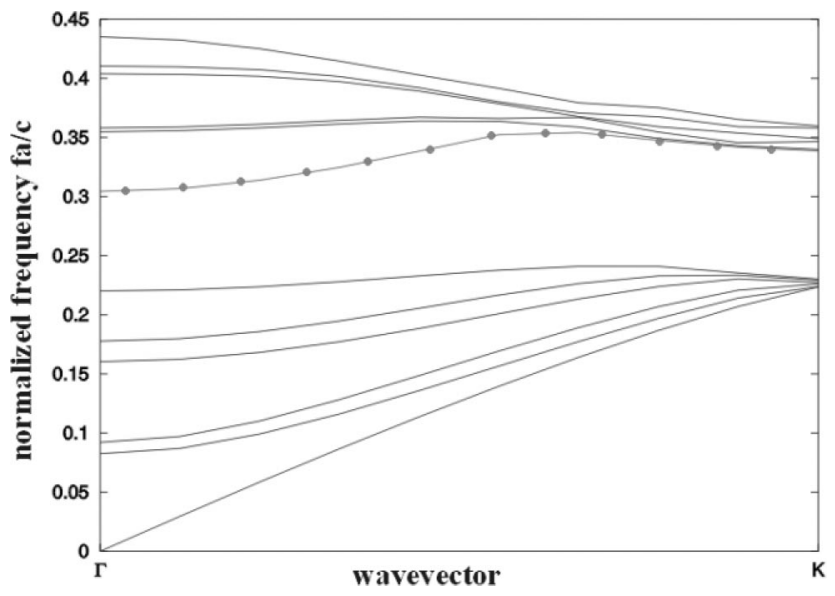

FIG. 4. Calculated band structure of the investigated 2D photonic crystal with the one-row line defect for certain wave vectors. The dotted line displays the frequencies of the propagating defect modes with frequencies inside the band gap. The straight lines mark the edges of the electromagnetic band gap.

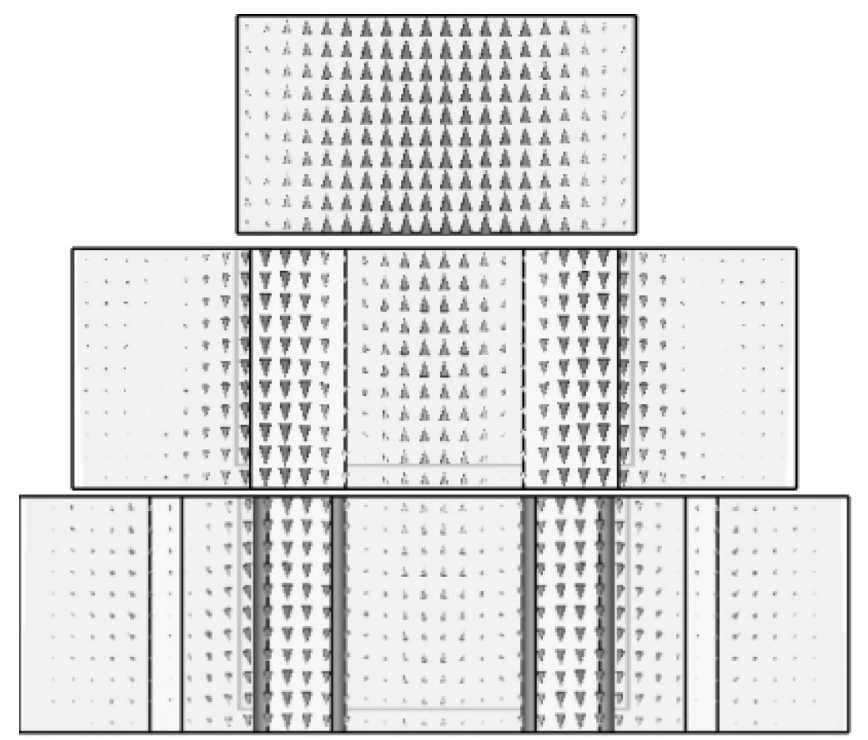

FIG. 5. View of the electric field distribution in (a) metal waveguide (b) transition region between metal and defect waveguide (c) one-row defect waveguide. The figure shows a cut through the structure perpendicular to the $x$ axis. We can clearly observe that the electric field has to undergo a change from zero field to maximum field in the transition area, which leads to bad coupling behavior.

electric fields in the metal waveguide have to be zero at the metal boundaries. At the corresponding position in the photonic crystal defect waveguide, the electric fields should have their first zero in order to achieve a good matching. In contrast, the field turns from a maximum in the middle of the defect to a minimum in the first line of dielectric rods. The results of the simulation addressing this field distribution can be seen in Fig. 5. There is a transition from vanishing electric field in the metal waveguide to a maximum of negative electric field at the $y$ value of the metal waveguide/defect waveguide boundary, which prohibits a good coupling between these two guiding parts. Reflection becomes significant and the transmission drops, as seen in our results.

To overcome this problem of mismatching we tried to find a line defect structure, where a matching between the waveguide parts is supported by geometrical considerations. A structure where three rows of rods have been removed from the 2D photonic crystal has been investigated. The transmission curve in the same frequency band as above is shown in Fig. 6, the structure is shown in the inset. Below and above the band gap we can observe again the highly structured transmission behavior related to the excitation of housing modes. However, compared with the case where only one line had been removed, the transmission curve shows a completely different behavior for frequencies within the band gap. We observe a broadband guidance of waves throughout the photonic band gap. Between 10.5 and 14.5 $\mathrm{GHz}$ the average transmission loss is $-0.07 \mathrm{~dB}$, and, apart from two minor dips in the transmission curve, the transmission loss never falls below $-1 \mathrm{~dB}$, which is a sign for a very good matching between both waveguide parts. From the analysis of the reflection coefficient the return loss was found to be around $-20 \mathrm{~dB}$ over this frequency range.

The discrepancy of coupling behavior between the three- 


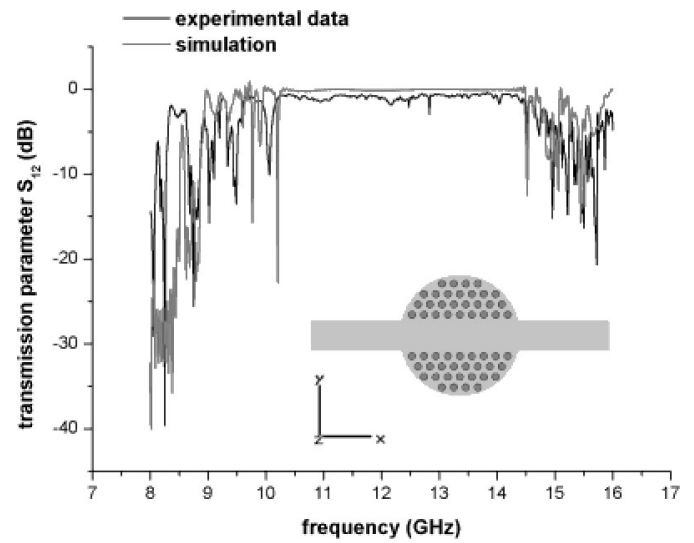

FIG. 6. Three row line defect in a 2D hexagonal photonic crystal: comparison between simulation and experimental result for the transmission parameter $S_{12}$. The structure under investigation is shown in the inset.

and the one-row line defect can be explained once again geometrically by examining the electric field of the traveling mode. The field distribution found in the simulation is shown in Fig. 7. The nodes of the metal waveguide mode are not in mismatch with defect geometry anymore, because of the larger defect width. The defect mode may have a minimum at the position of the first rods while additionally having a node at about the same $y$ position as the mode in the metal waveguide. The field can transit properly between those two waveguide types and a good coupling is achieved. A broadband guidance of waves over the whole photonic band gap is possible. A corresponding band structure calculation for the three-rows line defect can be seen in Fig. 8. A defect mode can be observed for any frequency inside the band gap (dotted lines in Fig. 8).

To extend the problem of controlled excitation of line defect modes to point defect modes, we have investigated an extended point defect in the photonic crystal. The structure

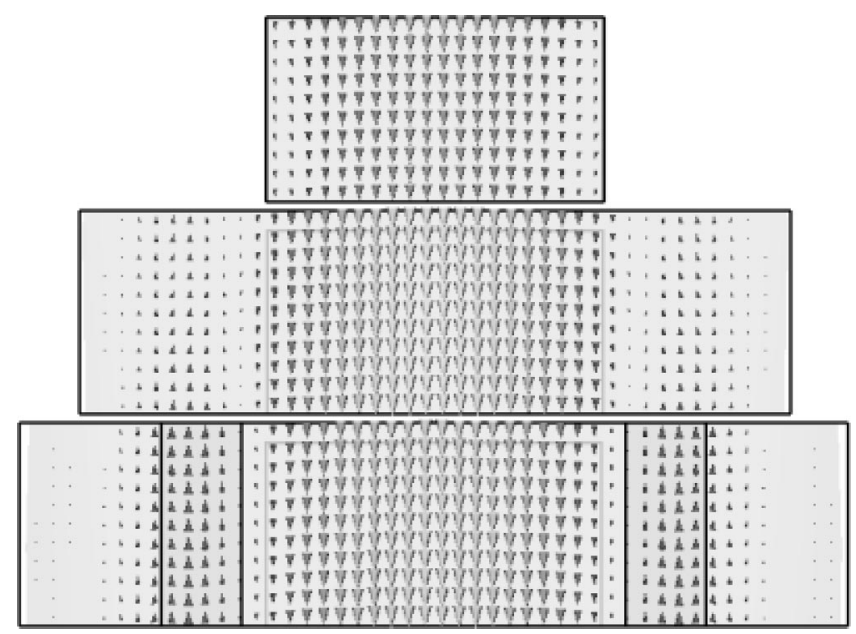

FIG. 7. Transverse view of the electric field distribution in (a) metal waveguide part, (b) transition area between metal and defect waveguide, (c) three row defect waveguide in the photonic crystal. The figure shows a cut through the structure perpendicular to the $x$ axis. We can observe that the chance the electric field has to undergo between metal waveguide part and line defect part is much smoother than for the one-row line defect shown in Fig. 4.

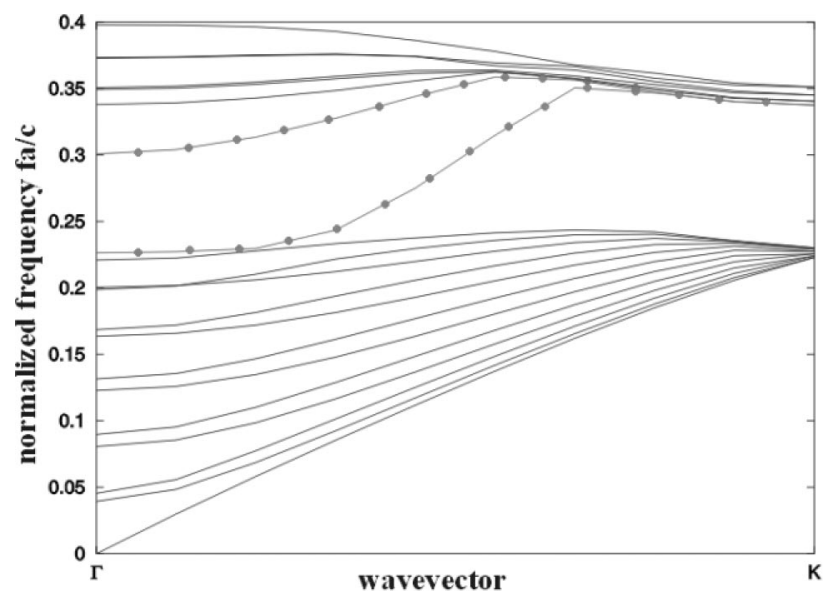

FIG. 8. Calculated band structure of the investigated 2D photonic crystal with the three-row line defect for certain wave vectors. The dotted lines display the frequencies of the propagating defect modes with frequencies inside the band gap. The straight lines mark the edges of the electromagnetic band gap.

can be seen in Fig. 9. We removed a number of rods in the middle of the photonic crystal structure to provide the defect. The coupling between the metal waveguide and the photonic crystal was provided by a line defect where three rows of rods have been left out. This structure allows a good coupling to the metal waveguide, as was explained before. The defect cavity was separated from the defect waveguide by two periods of the photonic crystal to allow for a localized mode.

\section{POINT DEFECT MODE EXCITATION}

According to conventional resonators, we expect that the strength of the coupling to the resonance will affect two parameters of the resonance peak: its height, resembling the insertion loss at resonance, and its width, resembling the loaded quality factor. For a strong coupling and without losses, we expect the peak maximum to approach $0 \mathrm{~dB}$ and a low loaded quality factor $Q_{l}$, while for a weak coupling the resonance peak should be little above the background noise and $Q_{l}$ should be nearly equal to the unloaded quality factor

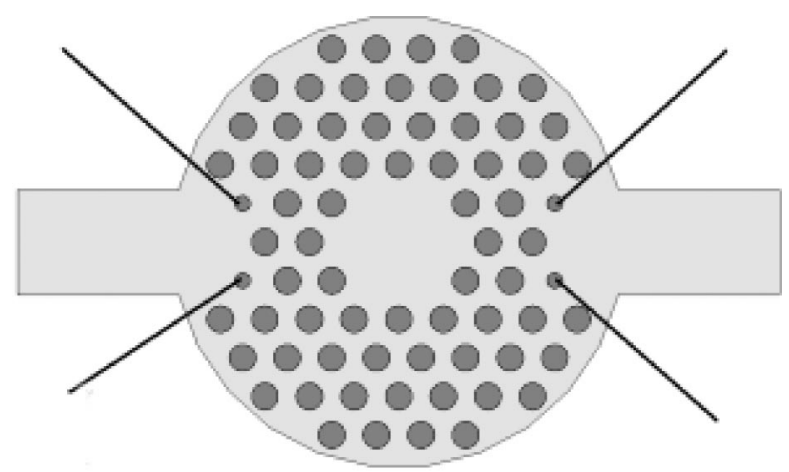

FIG. 9. By removing a number of rods in the middle of the EBG lattice, an extended defect was created. The marked rods have been varied between $r$ $=0 \mathrm{~mm}$ and $r=2.16 \mathrm{~mm}$ to change the strength of the coupling to the defect resonance. 


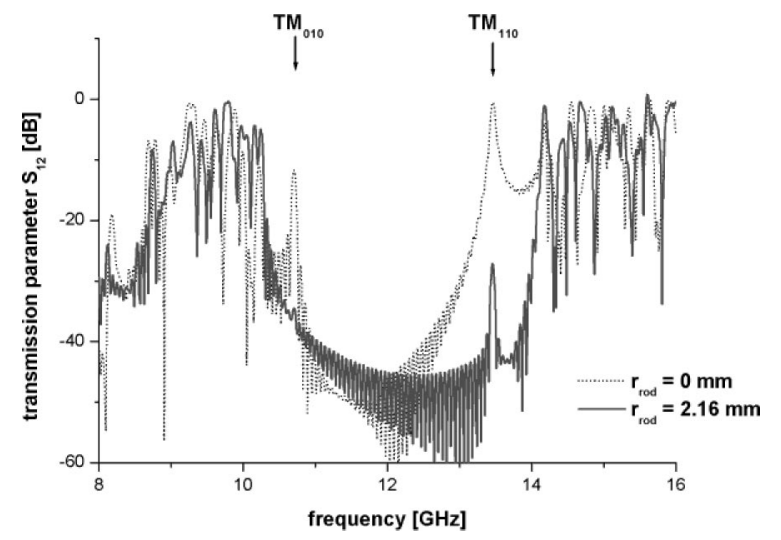

FIG. 10. Comparison of the behavior of the transmission peaks related to localized modes of the extended defect in our 2D photonic crystal for a varied radius of certain lattice elements (coupling rods).

$Q_{u}$. The value of $Q_{u}$ should be the same for every coupling strength, since it is determined only by the losses in the metal and dielectric parts of the resonator.

We started varying the photonic crystal lattice elements that connect the three row defect waveguide with the photonic crystal parts surrounding the defect and recorded the transmission curves, loaded, and unloaded quality factors of the resonances, and transmission peak heights. We found out that the coupling strength was highly sensitive to the radius of four particular rods (marked rods in Fig. 9). Those rods will be named "coupling rods." Therefore, we have varied
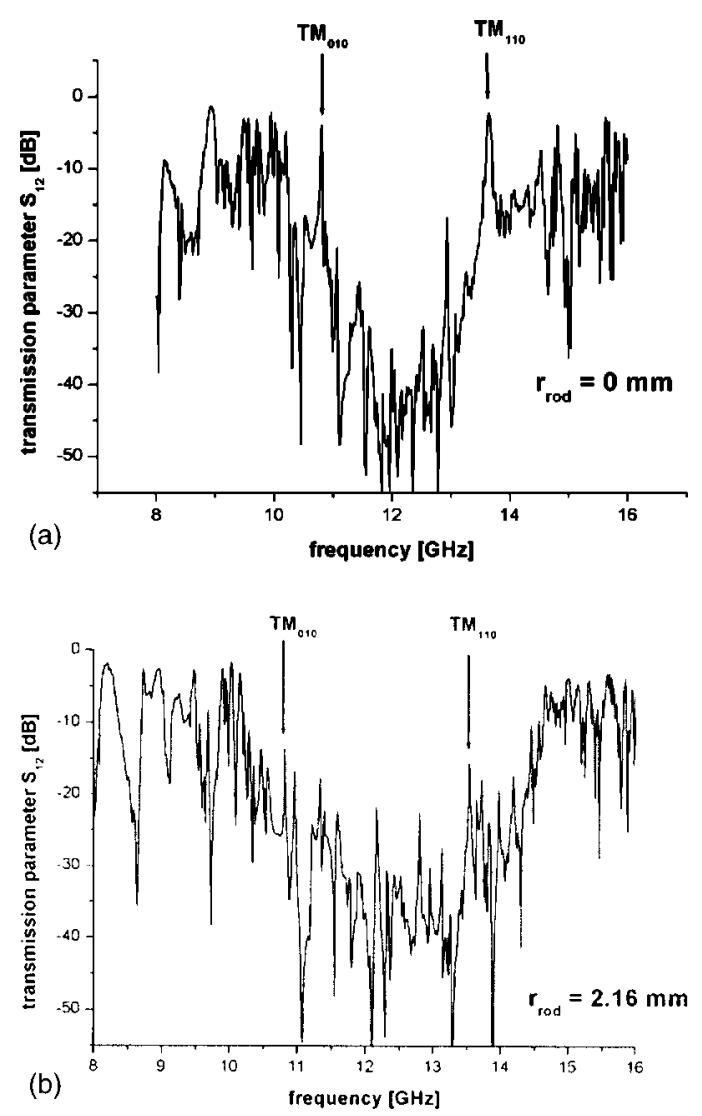

FIG. 11. Experimental results for the behavior of the transmission peaks related to the localized modes of the defect for different coupling rod radii.

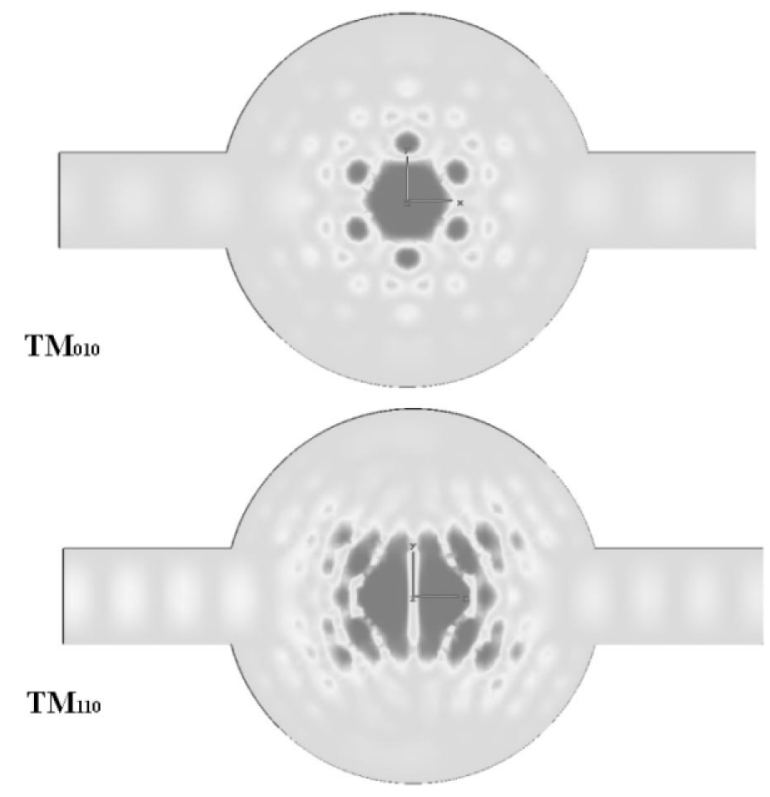

FIG. 12. Simulated electric field distribution (absolute value) of the resonant modes related to the observed transmission peaks. Mode frequencies are $f_{\text {TM010 }}=10.7 \mathrm{GHz}$ and $f_{\text {TM110 }}=13.45 \mathrm{GHz}$. Dielectric filling factors are $\kappa_{\mathrm{TM} 010}=0.59$ and $\kappa_{\mathrm{TM} 110}=0.52$ for a coupling rod radius $r=0 \mathrm{~mm}$.

the radius of the coupling rods, and recorded the scattering parameters for each of the rod radii both in simulation (Fig. 10) and experiment (Fig. 11). We have varied the rod radius in five steps between zero radius and normal radius. For better visibility, only two curves are shown in the figures. Apart from the known high structure density outside the band gap, the transmission curve exhibits two major resonant peaks with frequencies inside the band gap, those peaks are related to localized resonant modes that can be identified from the field simulation. The peak at $10.7 \mathrm{GHz}$ can be identified as the $\mathrm{TM}_{010}$ mode while the peak at $13.45 \mathrm{GHz}$ can be identified as the $\mathrm{TM}_{110}$ mode of the extended defect (see field distribution depicted in Fig. 12). Both resonances show the expected behavior, when the radii of the coupling rods are varied. For a small rod radius we observe a strong coupling to the resonance. The maximum $S_{12}$ value approaches $0 \mathrm{~dB}$, and we observe a very broad resonance peak for both modes. With an increasing rod radius the maximum transmission value in the resonance decreases, and the resonance peak almost vanishes in the background noise, when the rod radius has its normal value. The loaded quality factor of the resonances increases with rod radius, although the overall losses in metal and dielectric do not change. This increase in $Q_{l}$ must therefore arise from a weakened coupling strength.

The unloaded $Q$ factor due to metal losses in the cavity endplates for a $\mathrm{TM}_{\mathrm{nm} 0}(n \geqslant 0, m \geqslant 1)$ resonant mode with angular frequency $\omega$ can be calculated for a resonator with height $h$ and metal boundaries with a given surface resistance $R_{s}$ from the following equation:

$$
Q_{\mathrm{TM}}=\frac{G}{R_{s}}
$$

with

$$
G=\omega \mu_{0} h / 2 .
$$


The surface resistance of copper was calculated by

$$
R_{s}=\sqrt{\frac{\omega \mu}{2 \sigma}},
$$

assuming a dc conductivity of $\sigma=5.8 \times 10^{7} 1 /(\Omega \mathrm{m})$ for copper. For the resonant modes that we investigated, we received $Q_{\mathrm{TM} 010}=17.200$ and $Q_{\mathrm{TM} 110}=19.300$ from this formula. If we neglect the losses in the dielectric, our 3D simulation yields unloaded quality factors of 16.000 for the $\mathrm{TM}_{010}$ mode and 21.500 for the $\mathrm{TM}_{110}$ mode. We believe that these values coincide within the error of our numerical simulation.

The dielectric loss contribution to the unloaded quality factor was calculated by

$$
\frac{1}{Q_{u}^{d}}=\kappa \tan \delta,
$$

with $\kappa$ being determined to be 0.59 for the $\mathrm{TM}_{010}$ and 0.52 for the $\mathrm{TM}_{110}$ mode from our simulation. Taking into account dielectric losses, we received an overall unloaded $Q$ factor of 10.300 for the $\mathrm{TM}_{010}$ mode and 13.000 for the $\mathrm{TM}_{110}$ mode.

However, in the experiment we find that the unloaded $Q$ factors of both resonances have values of around 3000-4000 only. The reason for this discrepancy is currently not fully understood. We speculate that small deviations of the rod positions from their ideal positions in the hexagonal lattice as well as possible rod diameter and dielectric constant variations may have a strong effect on the maximum $Q$, similar to a distributed Bragg reflector microwave resonator reported in the literature. ${ }^{35}$ Experimental and numerical investigations to explain this discrepancy are currently in progress.

\section{CONCLUSION}

It has been shown that EBG line defect waveguides can be matched to external metal waveguides and that defect resonances can be excited in a very well defined manner. This finding represents an important step towards the practical use of EBG structures as microwave circuits. Possible applications are integrated oscillator circuits at millimeter wave frequencies, where transmission line resonators with defined insertion loss represent the frequency and phase noise determining circuit element. Our current research aiming towards such possible applications is to perform similar simulations and experiments for planar transmission lines rather than metal waveguides and for TM defect modes in dielectric slabs with 2D EBG lattice rather than 2D EBG lattices terminated by metal walls. We hope that higher quality factors can be achieved if microfabrication techniques with smaller tolerances will be employed. Our efforts may help to pave the wave to a novel low-loss integrated circuit technology with possible impact from the millimeter wave to the terahertz range.

\section{ACKNOWLEDGMENTS}

The authors would like to thank D. Parkot for the band structure calculations. This work has been funded in part by the European Commission in the framework of EU-Project "FOAMS."

${ }^{1}$ E. Yablonovitch, Phys. Rev. Lett. 58, 2059 (1987).

${ }^{2}$ S. John, Phys. Rev. Lett. 58, 2486 (1987).

${ }^{3}$ C. T. Chan, K. M. Ho, and C. M. Soukoulis, Europhys. Lett. 16, 563 (1992).

${ }^{4}$ J. D. Joannopoulos, R. D. Meade, and J. N. Winn, Photonic Crystals (Princeton University Press, Princeton, NJ, 1995).

${ }^{5}$ R. D. Meade, K. D. Brommer, A. M. Rappe, and J. D. Joannopoulos, Phys. Rev. B 44, 13772 (1991).

${ }^{6}$ E. Yablonovitch, T. J. Gmitter, R. D. Meade, A. M. Rappe, K. D. Brommer, and J. D. Joannopoulos, Phys. Rev. Lett. 67, 3380 (1991).

${ }^{7}$ V. Kuzmiak and A. A. Maradudin, Phys. Rev. B 57, 15242 (1998).

${ }^{8}$ V. Kuzmiak and A. A. Maradudin, Phys. Rev. B 61, 10750 (2000).

${ }^{9}$ K. Busch, N. Vats, S. John, and B. C. Sanders, Phys. Rev. E 62, 4251 (2000).

${ }^{10}$ P. R. Villeneuve, S. Fan, and J. D. Joannopoulos, Phys. Rev. B 54, 7837 (1996).

${ }^{11}$ O. Painter, J. Vuckovic, and A. Scherer, J. Opt. Soc. Am. B 16, 275 (1999).

${ }^{12}$ E. Ozbay, B. Temelkuran, M. Sigalas, G. Tuttle, C. M. Soukoulis, and K. M. Ho, Appl. Phys. Lett. 69, 3797 (1996).

${ }^{13}$ M. Agio, E. Lidorikis, and C. M. Soukoulis, J. Opt. Soc. Am. B 17, 2037 (2000).

${ }^{14}$ R. K. Lee, O. Painter, B. Kitzke, A. Scherer, and A. Yarif, J. Opt. Soc. Am. B 17, 629 (2000).

${ }^{15}$ T. Ueta, K. Ohtaka, N. Kawai, and K. Sakoda, J. Appl. Phys. 84, 6299 (1998).

${ }^{16}$ M. Bayindir, E. Ozbay, B. Temelkuran, M. M. Sigalas, C. M. Soukoulis, R. Biswas, and K. M. Ho, Phys. Rev. B 63, 081107 (2001).

${ }^{17}$ M. Bayindir, B. Temelkuran, and E. Ozbay, Appl. Phys. Lett. 77, 3902 (2000).

${ }^{18}$ A. Mekis, J. C. Chen, I. Kurland, S. Fan, P. R. Villeneuve, and J. D. Joannopoulos, Phys. Rev. Lett. 77, 3787 (1996).

${ }^{19}$ A. Mekis, S. Fan, and J. D. Joannopoulos, Phys. Rev. B 58, 4809 (1998).

${ }^{20} \mathrm{~S}$. Lin, E. Chow, V. Hietala, P. R. Villeneuve, and J. D. Joannopoulos, Science 282, 5387 (2000).

${ }^{21}$ M. Tokushima, H. Kosaka, A. Tomita, and H. Yamada, Appl. Phys. Lett. 76, 952 (2000).

${ }^{22}$ S. Yamada, T. Koyama, Y. Katayama, N. Ikeda, Y. Sugimoto, K. Asakawa, N. Kawai, and K. Inoue, J. Appl. Phys. 89, 855 (2001).

${ }^{23}$ C. J. M. Smith, R. M. de la Rue, M. Rattier, S. Olivier, H. Benisty, C. Weisbuch, T. F. Krauss, R. Houdre, and U. Oesterle, Appl. Phys. Lett. 78, 1487 (2001).

${ }^{24}$ A. Yarif, Y. Xu, R. K. Lee, and A. Scherer, Opt. Lett. 24, 711 (1999).

${ }^{25}$ M. Qiu, K. Azizi, A. Karlsson, M. Swillo, and B. Jaskorzynska, Phys. Rev. B 64, 155113 (2001).

${ }^{26}$ W. M. Robertson, G. Arjavalingam, R. D. Meade, K. D. Brommer, A. M. Rappe, and J. D. Joannoupoulos, Phys. Rev. Lett. 68, 2023 (1992).

${ }^{27}$ S. L. McCall, P. M. Platzmann, R. Dalichaouch, D. Smith, and S. Schultz, Phys. Rev. Lett. 67, 2017 (1991).

${ }^{28}$ C. M. Anderson and K. P. Giapis, Phys. Rev. Lett. 77, 2949 (1996).

${ }^{29}$ P. R. Villeneuve and M. Piché, Phys. Rev. B 46, 4969 (1992).

${ }^{30}$ M. Plihal and A. A. Maradudin, Phys. Rev. B 44, 8565 (1991).

${ }^{31}$ D. Cassagne, C. Jouanin, and D. Bertho, Phys. Rev. B 53, 7134 (1996).

${ }^{32}$ T. Sondergaard and K. H. Dridi, Phys. Rev. B 61, 15688 (2001).

${ }^{33}$ M. Notomi, K. Yamada, A. Shinya, J. Takahashi, C. Takahashi, and I. Yokohama, Phys. Rev. Lett. 87, 253902 (2001).

${ }^{34}$ T. Sondergaard, Opt. Commun. 203, 263 (2002).

${ }^{35}$ C. A. Flory and H. L. Ko, Proceedings of the 1997 IEEE International Frequency Control Symposium (1997), p. 994. 